مجلة جامعة /لملك عبدالعنيز: الاقتصاد الإسلامي، م32 ع3، ص ص: 137-150 (محرم 1441/ أكتوبر 2019) DOI:10.4197/Islec.32-3.8

\title{
منهجية البناء المنهجي لعلم الاقتصهاد الإسلامي
}

\author{
عبد الرحيم عبدالحميد السـاعاتي \\ باحث - معهل الاقتصاد الإسلامي \\ جامعة الملك عبدالعزيز - جدة - المملكة العببية السعودية المادية
}

المستخلص. أدى غياب مفهوم المنهجية (methodology)، الذي هو جزء من فلسفة المعرفة

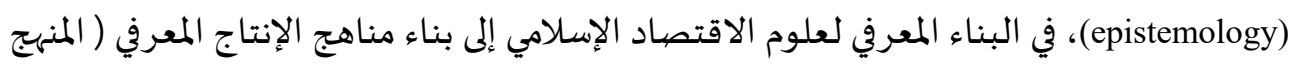

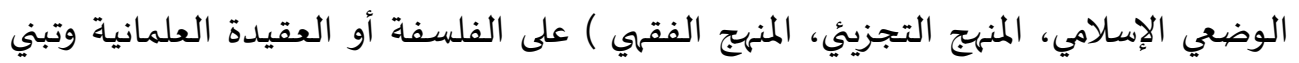

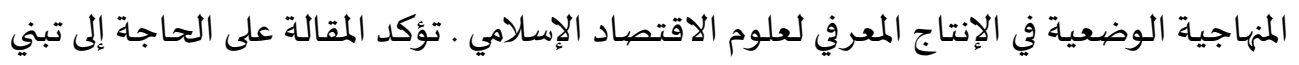

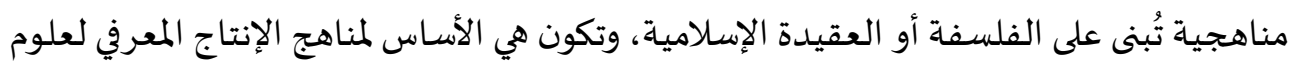
الاقتصاد الإسلامي وتضع تصورًا لمناهجية البناء المنهجي لعلم الاقتصاد الإسلامي. الكلمات الدَّلة: المناهجياة، المنهجية ، فلسفة المعرفة، المنظور الاقتصادي تصنيف B410, B590 تصنيف H21 


\section{المقدمة}

والمنهجية في الأدبيات الغربية تقع في حقل علم فلسفة المعرفة أو الأبستمولوجيا، وسوف أستخدم لفظ المنهاجية بدل المنهجية وذلك لشيوع استخدام كلمة المنهجية ويقصد المبدا بها الطريقة أو منهج البحث. إن منهاجية دراسة علم

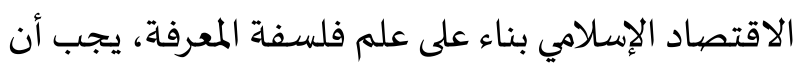

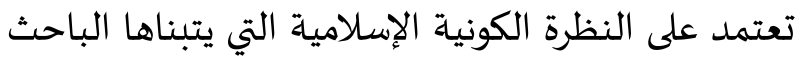
والمجتمع العلمي، وهذه تكون مبنية على الفلسفة أو

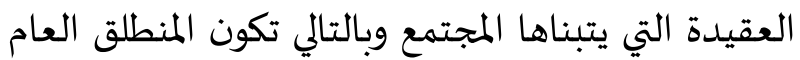
للمعرفة، والمنهاجية الإسلامية بما في ذلك المعرفة الاقتصادية التي تُعد من نتاج فلسفة المعرفة الإسلامية، أو (الابستمولوجيا الإسلامية)، وحيث إن المهناجية تحددها فلسفة المعرفة

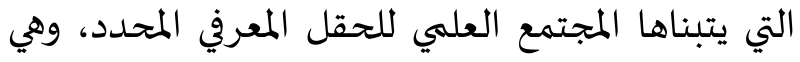
جزء لا ينفصل عن الفلسفة أو العقيدة التي تسود في

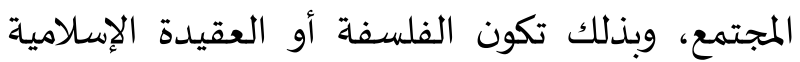

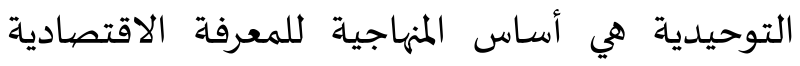

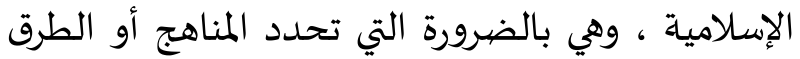

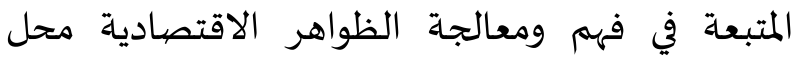
الدراسة في المجتمع المسلم، وتؤكد هذه القاعدة الدراسات التي تبين استحالة التطرق للأيديولوجية دون فلسفة أو الو المالي عقيدة تبنى عليها (Karl [1929).

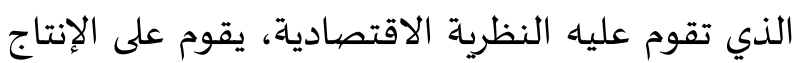

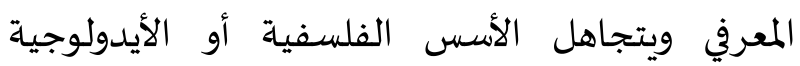

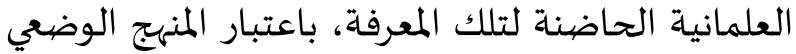
هو المنهج الأوحد لدراسة الاقتصاد وهي حقيقة مسلم بها في الفكر الاقتصادي السائد، وبناءً على هذا المبدأ كان

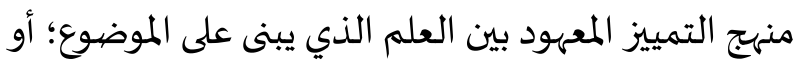
الواقع المحسوس وبين العقيدة أو الأيديولوجية

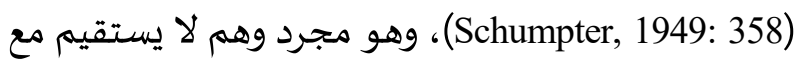
الواقع (Heilbroner, 1996:47).
لم تكن هناك حاجة للتفريق بين المنهجية والمناهج في الإنتاج المعرفي في الحضارة الإسلامية، ذلك أن الحضارة الإسلامية وعلومها بنيت على الفلسفة أو العقيدة الإسلامية، وحتي حينما بدأت الفلسفات الاغريقية أو الهندية أو الرومانية

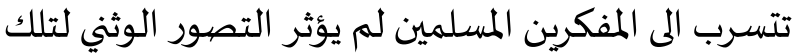
الفلسفات على العقيدة الإسلامية التوحيدية، ولكن علم الاقتصاد ومناهج دراسته ولد في أحضان الفلسفة المادية العلمانية فكان المنهج الوضعي الذي يستبعد القيم والأخلاق

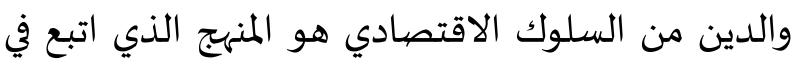
تأسيس علم الاقتصاد بسبب اختلاف الفلسفة أو العقيدة التي تبني عليها فلسفة المعرفة بين الدين العلماني والدين

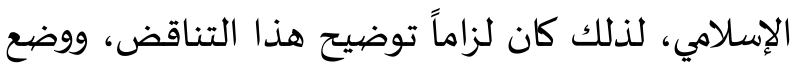

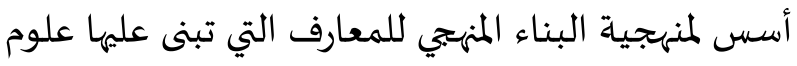
الاقتصاد الإسلامي التي يجب أن تؤسس على عقيدة التوحيد

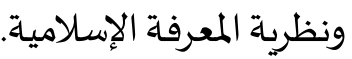

\section{1- المنهجية ومناهج المعرفة الاقتصادية الإسلامية} لا تفرق الأدبيات العربية بين المنهجية (methodology) وبين المنهج (method)، حيث يشتق مفهوم المنهجية من

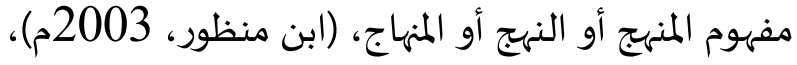

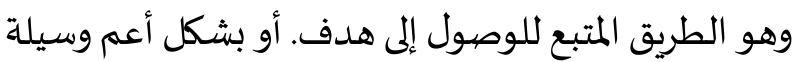
محددة توصل إلى غاية معينة، إذا كانت المناهج (methods) هي طرق البحث وإجراءاته، فإن المنهجية (methodology)

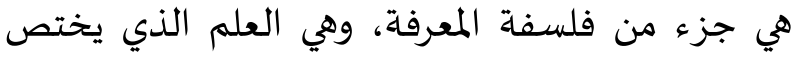
بدراسة طرق البحث العلمي ومناهجه وإجراءاته، وهي تتضمن بالضرورة التصور والتخطيط المسبق والرؤية الكلية للموضوع محل البحث، والأهداف المعرفية منه،

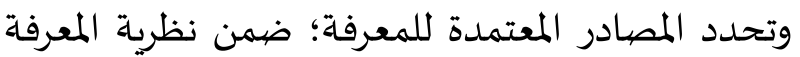
ووسائل التحقق من صحة المعرفة التي تم استنتاجها. 
(Dictionary (2009)، وهذا هو الدور الذي يجب أن يقوم به الدين كما يراه ماكس وبر (Max Weber, 1993) فالسلوك الديني في نظره لا يكون اجتماعياً إذا اقتصر على التأمل، أو

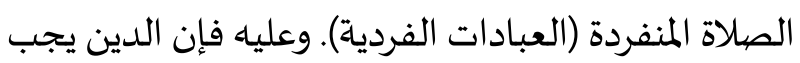
أن يغير سلوك الإنسان لتتفق مع الأهداف والقيم التي ينادي بها الدين، لذلك فإن المنهاجية التي يُبنى عليها علم

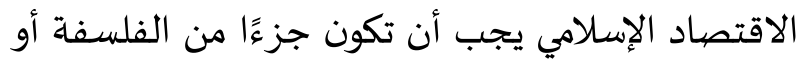
التصورات الكلية الإسلامية، ويجب على المعتقدات العلمية أو الفرضيات الأساسية لعلم الاقتصاد الإسلامي

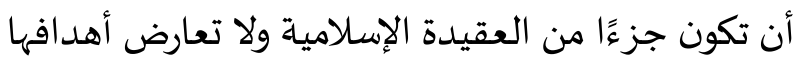

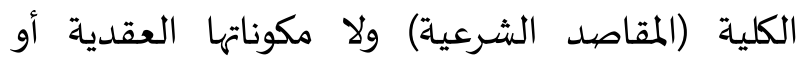
ضوابطها للسلوك البشري عامة، وللسلوك الاقتصادي للوحدات الاقتصادية الذي هو موضوع علم الاقتصاد. إن منهاجية دراسة علم الاقتصياد الإسلامي يجب أن

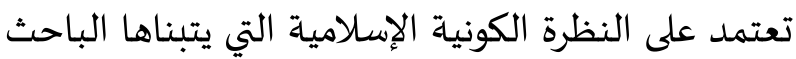

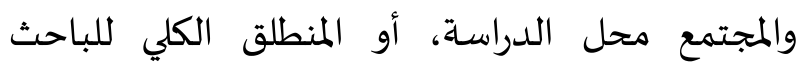
والمجتمع. والمههاجية الإسلامية للمعرفة تعتبر الوحي أو النقل مصيدرًا مهمًا للمعرفة الإنسانية، وتعتبره المصيدر المطلق للحقيقة، فتكون من العوامل المؤثرة في السلوك الاقتصادي وهو موضوع علم الاقتصاد، وكذلك مكونات العقيدة التوحيدية التي منها: الإيمان بالله وملائكته وكتبه ورسله واليوم الآخر والجنة والنار والحلال والحرام والقضاء والقدر والحساب والعقاب. وهذه المنهاجية تعتمد

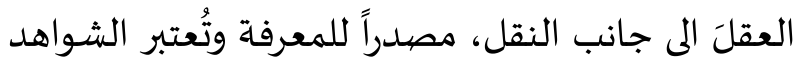
الحسية أو المشاهدات والاستنتاج المنطقي مصدرًا للمعرفة الإنسانية التي يمكن أن يدركها الإنسان، ولكن الفلسفة أو العقيدة الإسلامية تقتضي الاعتقاد بمحدودية العقل في إدراك كل شيء وقصور رشده، وبعدم منطقية السلوك الإنساني وتحيُّزه أحياناً، وهذا يتطابق مع مع ما
باستقراء الأدبيات باللغتين العربية والإنجليزية عن المنهاجية في الاقتصاد الإسلامي يمكن القول أنه لا توجد دراسة عن منهاجية الإنتاج المعرفي لعلم الاقتصاد الإسلامي، ووجد أسلم حنيف (2012م) أن المناهج المتبعة في التنظير لعلم الاقتصاد الإسلامي، تميل إلى التكامل والتصالح مع المنهاجية المتبعة في التنظير في علم الاقتصياد الرأسمالي، حيث عملت مناهج دراسة الاقتصياد الهميهاد الإسلامي على إدخال القيم والضوابط الشرعية على النظريات الرأسمالية دون الإخلال ببنيتها الأساسية، ومبررهم في ذلك أنها يجب عدم تجاهل التطور الكبير في علم الاقتصياد ونظرياته، بل يجب الإفادة منه في بناء علم الاقتصاد الإسلامي، وشذ عن ذلك شودري الذي يرى أن المنهجية هي الفلسفة التي بُني عليها علم الاقتصاد التقليدي وهي لا تصلح أن تكون أساسًا للبناء المعرفي لعلم الاقتصاد الإسلامي ونحتاج في نظره إلى منهجية حقيقية (توحيدية) تأخذ الحقيقة المادية والروحية في تأسيسها للاقتصاد الإسلامي. وكذلك يرى أسعد الزمان رفض الاقتصاد النيوكلاسيكي السائد بسبب تناقض مكونه المعرفي مع مكون الاقتصاد الإسالامي، وبسبب اختلاف النظرة الكونية وتفسير مبررات الوجود في كلِّ من النظرة الكونية الإسلامية والرأسمالية.

2-منهاجية البناء المنه-جي لعلم الاقتصياد الإسلامي: إذا كانت منهاجية البحث هي الفلسفة التي ينطلق منها المنظور والقناعات والقيم العلمية لذلك الحقل العلمي (Paradigms)، فإنه يجب على هذه القناعات أن تكون جزءًا لا يتجزأ من القناعات والقيم والتصورات التي ينطلق منها الباحث، ومصدر هذه القناعات يكون هو الدين. والدين يمكن تعريفه بأنه: (مبادئ وتفسيرات أو نشاطات The American Heritage ( تمارس بناءً على إخلاص إرادي)، 
فيها المسلم وغير المسلم، ويكون الفرق في تأثير القيم والضوابط الشرعية على هذا السلوك. وهذا التغير يمكن استيعابه في التحليل الاقتصادي التقليدي الذي تسمح الطرق الرياضية والقياسية فيه بوضع قيود على السلوك الاقتصادي والحصول على السلوك الأمثل تحت هذه القيود، وبذلك يشترك علم الاقتصاد الإسلامي مع علم الاقتصاد التقليدي في الاعتقاد بوجود قوانين حتمية تحكم السلوك الاقتصادي للمسلم، أما بالنسبة لتدخل الدولة في الاقتصاد الإسلامي فإناه وكما يتيح علم الاقتصاد التقليدي في مجال السياسة الاقتصادية تدخل الدولة في الاقتصاد ووضع قيود على السلوك الاقتصادي للوحدات الاقتصادية لتحقيق أهداف كلية يتبناها المجتمع، كذلك في الاقتصاد الإسلامي يمكن للدولة التدخل في توجياء الاقتصاد، حيث يمكن اعتبار الزكاة نوعًا من الضرائب، وتحقيق التكافل الاجتماعي نوعًا من شبكات الأمان الاجتماعي التي تديرها الدولة، وتحريم الربا يكون ضمن السياسة النقدية في تبني الفائدة الصفرية، والفائدة تسمى لـ معدل الربح في المرابحة أو التورق المنظم، وهو قيمة الفرصة البديلة لاستخدام النقود، أو القيمة الزمنية للنقود في عقود البيوع الآجلة، أما القمار والاتجار في المخاطر في التأمين فهو لازال من مكونات الاقتصاد الإسلامي في هذا المنهج وينفذ تحت مسمى التأمين التكافلي، أو مسهى بيع العربون في عقود الخيارات في

$$
\text { المشتقات المالية الإسلامية. }
$$

يعتقد أتباع مدرسة التحليل الاقتصادي الوضعي الإسلامي، إن محل علم الاقتصاد الإسلامي؛ أو موضيوعاء

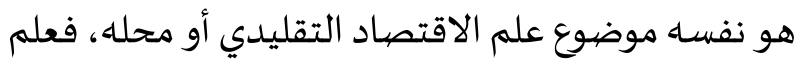
الاقتصاد الإسلامي يدرس الموارد الاقتصادية النادرة وغير الكافية لتلبية الرغبات غير المتناهية للأفراد في المجتمع
توصلت إليه الدراسات التطبيقية لعلم الاقتصاد السلوكي. لذلك يحتاج البناء المعرفي لعلم الاقتصياد الإسلامي إلى علوم النقل أو الوحي لوضيع معايير الرشد الذي تطلع بها علوم الفقاه، والتنظير للسلوك المنطقي من المنظور الإسلاهي، ووضع الأهداف الكلية للمجتمع المسلم والسياسات الاقتصادية التي تحقق تلك الأهداف، لذلك تعتمد المنهاجية الإسلامية العقل والمشاهدات لاكتشاف السنن التي تسير عليها الظواهر الاقتصادية لفهمها وتوجيهها وتسخيرها لتحقيق وظيفة الاستخلاف، كما تعتمد على

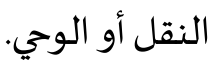

\section{3-مناهج المعرفة الاقتصادية الإسلامية وأسسها:} يمكن التميز بين مناهج الإنتاج المعرفي للاقتصاد

$$
\text { الإسلامي بين المناهج التالية: }
$$

أ- منهج التحليل الاقتصهادي الإسالامي الوضسي: يقوم هذا المنهج بوضع الأطر النظرية للسلوك الاقتصادي في المجتمع الإسلامي، وفي هذا المنهج يتم البناء النظري لعلم الاقتصاد الإسلامي على القيم العلمية نفسها والافتراضات الأساسية لعلم الاقتصاد الرأسمالي، على الرغم من تناقض المنظور والفلسفة أو العقيدة العلمانية المولّدّة لتلك القيم العلمية والمنظور الاقتصادي (Paradigms)، للمنظور الإسلامي، فكانت نظريات السلوك الاقتصادي للمجتمع المسلم هي النظريات الغربية نفسها مع إدخال القيم أو الضوابط الشرعية أو المؤسسات المسات الاقتصادية مثل: الوقف والزكاة وتحريم الربا والقمار كمحددات على السلوك الأمثل في مفهوم النظرية النيوكلاسيكية أو الكينزية، في هذا المنهج تعتبر الدوافع والحوافز ومحركات السلوك الاقتصيادي للمسلم هي نفسها في المجتمع الرأسمالي ، فعلم الاقتصاد الإسلامي

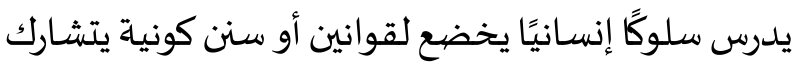


على الودائع والقروض وتحسين الجدارة الائتمانية، كما يدخل في هذا المنهج استخدام الهندسة المالية في إعادة إنتاج المنتجات المالية الغربية في مظهر وشكل ومسمى إسلامي عن طريق الحيل والمخارج الفقهية. إن هذا المنهج مهم لأناه يعالج قضايا تطبيقياة وعملية تواجاه العاملين في الصناعة المالية الإسلامية، وإن تعارض مع المبادئ الأساسية للنظام الاقتصادي الإسلامي. يجذب هذا المنهج الكُتَّاب الغربيين بحكم تمكنهم في الجوانب الفنية في النظام المالي، وعدم اطلاعهه واهتمامهه بالقضيايا الكلية التي يهتم بها الاقتصاد الإسلامي، كما يهم هذا المنهج طلاب الدراسات العليا في الجامعات الغربية، حيث يجد هذا المنهج قبولاً من المشرفين على أطروحاتهم، كما ترحب بمنتجات هذ المنهج المجلات العلمية الغربية التي تهتم بالأبحاث الكمية والموضوعية حيث تعالج هذه الدراسات قضايا فنية لا مكان فيها للقيم الأخلاقية أو الدينية.

$$
\text { ج - المناهج الفقهية: }
$$

تاريخياً نمت الدراسات في الاقتصاد الإسلامي عندما وجد المفكرون المسلمون تعارضًا بين قيم وأدوات النظام الاقتصادي الرأسمالي والضوابط الشرعية في المعاملات خاصة في القطاع المالي، لذلك عمل الفقهاء على دراسة المعاملات المالية في النظام الاقتصادي الرأسمالي ومدى المئي تعارضها مع الضوابط الشرعية لإيجاد بدائل لها، فكان الربا وتغلغله في النظام الاقتصادي والمالي محفزاً للمفكرين الإسلاميين للبحث عن بديل لتجنب الربا وتقديم شواهد لتميز النظام الإسلامي عن النظام الرأسمالي، فتركزت الكتابات على الربا وأحكامه وبدائله الإسلامية بسبب مركزية الفائدة أو الربا في هيكلية النظام الرأسمالي والإجماع على تحريماء بنصوص قطعية الدلالة وقطعية الثبوت، وكذلك تركزت الكتابات حول الزكاة باعتبارها ركنًا
المسلم، أي أن محل علم الاقتصاد الإسلامي هو محل علم الاقتصاد التقليدي نفسـه ، وهدف النشاط الاقتصادي في المجتمع المسلم هو تحقيق تلك الرغبات لتحقيق الرفاه البشري، وتسمح النظرية الاقتصادية الغربية بتبني معنى مرن للرفاه ليشمل الجوانب الروحية والأخلاقية، بذلك يمكن لعلم الاقتصاد الإسلامي أن يتبنى معنى الرفاه الذي يشمل الرفاه المادي والمعنوي، لذلك كان هدف الاقتصاد الإسلامي في هذا المنهج تحقيق الرفاه المادي وتحقيق الأخوة الإنسانية والعدالة الاجتماعية، وحرمة الحياة والخصيوصية، وفي هذا المنهج كان موضوع علم الاقتصاد الإسلامي دراسـة المتغيرات الاقتصادية التي يمكن أن تستوعب نماذجه الرياضية وتأثير السلوك الاقتصادي بالقيم الإسلامية والعوامل الأخلاقية والنفسية والاجتماعية والسياسية والديموغرافية والتاريخية التي قد تؤخذ في الاعتبار في وضع السياسات الاقتصادية التي تحقق الرفاه المادي والمعنوي. ب- المنهج التطبيقي الجزئي: هذا المنهج مبني على المنهجية الوضعية وعلى قيمها العلمية، وهو يقوم على معالجة فنية جزئية لقضايا تهم

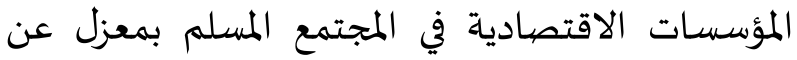
مبادئ وأسس الاقتصاد والتمويل الإسلامي، وهو يهدف إلى تطويع المؤسسات المالية والاقتصادية الإسلامية والأدوات والعقود المستخدمة لتتوافق مع المنهج الرأسمالي في

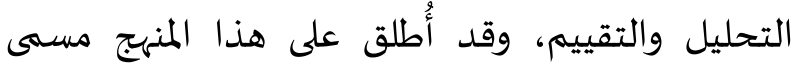
(Islamic Economics Light) مؤسسات التمويل أو التأمين أو المصارف الإسلامية ويستخدم في ذلك النماذج نفسها والطرق المستخدمة والأدوات في دراسـة المؤسسات المالية والاقتصادية الغربية مثل: الكفاءة والتكلفة وإدارة المخاطر والتحوط والتأمين 
الحكم الشرعي، وقد أدى هذا إلى اختلاف الأحكام الشرعية

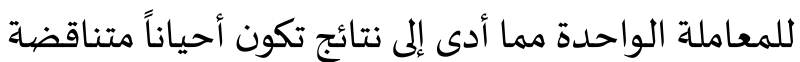
(الساعاتي، 2012م)، فالكُتَّاب الفقهاء في الاقتصاد الإداد

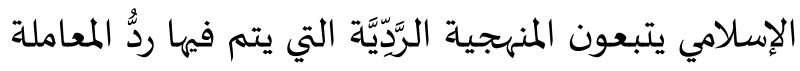

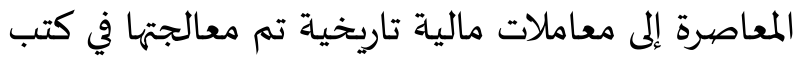
الفقاه من خلال تفتيت هذه المعاملة المعاصرة إلى أجزاء يسيرة، أو إلى أقل عناصر تتألف منها ومعاملها جزءًا طبقا لأداة التناظر والتشابه، وبذلك يتم المحافظة على التراث الفقهي. أو يتبعون المنهجية المقاصدية، وهو منهج كلي حيث تتم الاستعانة ببعض التراث الفقهي بعد تعديله وتنقيحه في فهم مقاصد بعض المعاملات المالية المعاصرة، وعملية البحث الشرعي لهذه المعاملات يجب أن تسير وفقاً للطريقة الكلية، التي تقوم على مسلمة مؤداها أن الن المعادية المعاملات الاقتصادية أو المالية المعاصرة معاملات مستحدثة لم تكن معروفة لدى الفقهاء، لذلك يتم

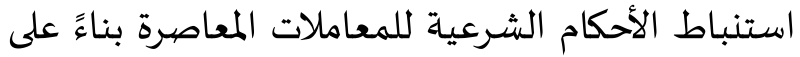
نوعين من المقدمات: مقدمة كلية كبرى (الكلية الشرعية أو المقصد الشرعي) ، ومقدمة كلية صغرى (عقلية أو تجريبية)، وقد أدى الانقسام المنهجي إلى تناقضيات فكرية وتنظيرات متباينة، وإلى عدم اتفاق الفقهاء على الحكم الشرعي

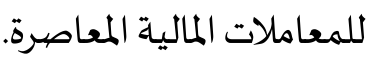

4- الحاجة إلى مراجعة منهجيات ومناهج الإنتاج المعرفي

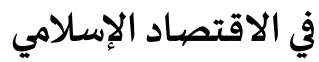
أ- من أسباب مفارقات مناهج الإنتاج المعرفي الإسلامي

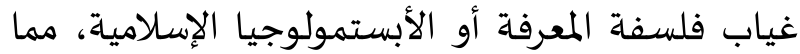
أدى إلى عدم الاهتمام بحقل فلسفة المعرفة أو الأبستمولوجيا في بناء نظرية المعرفة الإسلامية في حقل الاقتصاد، وقد المدادي أدى ذلك إلى تبني الكتابات في مناهج الإنتاج المعرفي الإسلامي لمفاهيم اقتصادية مبنية على العقيدة العلمانية التي نتج
من أركان الإسلام، وكان دور المنهج التحليلي في الاقتصاد

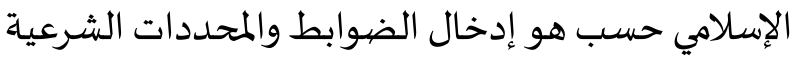
التي توصل لها الفقهاء على النماذج الاقتصادية الرأسمالية لبناء النظام المالي الإسلامي. إن الدور الذي قام بها المنهج الفقهي في تحرير المعاملات الاقتصادية والمالية من المخالفات الشرعية دور مهم كحل

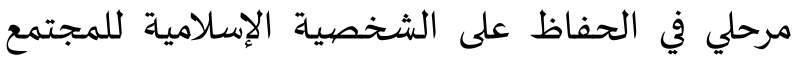
المسلم، ولكنه كان ضمن المنظور الاقتصادي الرأسمالي وضمن قيمه العلمية ومنظوره الاقتصادي ،فوجد النظام

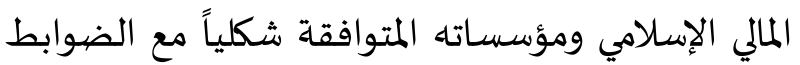
الشرعية أهها تحقق وظائف النظام المالي الرأسمالي، ولكن المنهج الفقهي الذي عالج قضايا جزئية في النظام الرأسمالي لم يؤد الى تحقيق المقاصد الشرعية للنظام الاقتصادي الإسلامي في حفظ المال وتداوله وعدم تركزه عند الأغنياء، وتحقيق وظيفة الاستخلاف على الموارد الاقتصادية التي

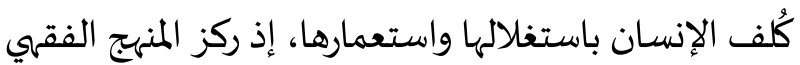
على إيجاد بدائل للمعاملات المخالفة للشريعة، وعلى المعالجات الجزئية لمكونات النظام الاقتصادي الرأسمالي واقتصر دور الاقتصاديين المسلمين حسب هذا المنهج على دراسة تأثير الضوابط الفقهية على النماذج الاقتصادية

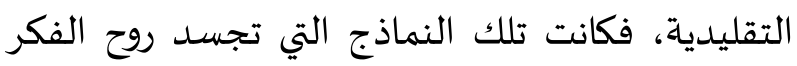
الاقتصادي الرأسمالي هي الأسساس في التحليل الاقتصادي الإسلامي، لذلك لم يختلف التحليل الاقتصادي الإسلامي عن التحليل الاقتصادي النيوكلاسيكي في التحليل الجزئي، أو الكينزي في تحليله الكلي، فبذلك افتقد التحليل الاقتصادي الإسلامي أو علم الاقتصاد الإسلامي الأصالة إذل لم يجرؤ على الخروج من جُبَّة علم الاقتصاد التقليدي. تنقسم المدرسة الفقهية لدراسة الاقتصاد الإسلامي إلى

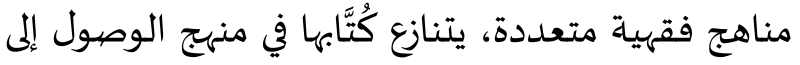


ج- تناقض المنهج الوضيعي مع العقيدة الإسلامية: على الم الميدة

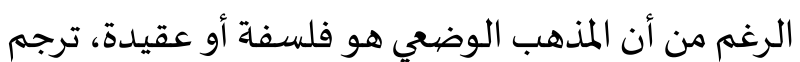

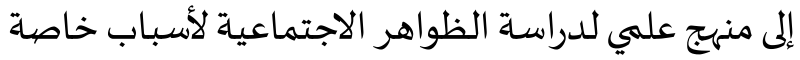
بالمجتمع الأوروبي في عهد التنوير، إذ كان من مبررات تبنيه الرغبة في تجاوز المعتقدات الدينية، وفرضيات علم ما وراء الطبيعة، فظهر الاقتصاد الوضعي لينظر لما هو كائن ويتميّز بذلك عن الاقتصاد السياسي التقليدي الذي ينظر لما يجب أن يكون، فكان موضوع علم الاقتصاد ينحصر في

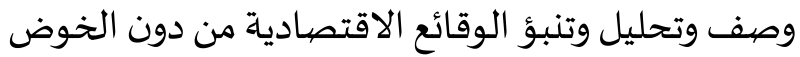
في الغايات، حيث يودي المنهج الوضعي في دراسة الظواهر الاقتصادية الى حصر موضوع علم الاقتصاد في اكتشاف القوانين الطبيعية التي تحكم العلاقات الاقتصادية مثلما تحكم القوانين الطبيعية في علم الفيزياء، وحيث إن هذه القوانين لها غاية كامنة تتحدد في تحقيق التوازن في حالة الخروج عنه بسبب تغير أحد المتغيرات، إذ أن المنهج الوضعي في دراسة العلاقة بين المتغيرات في الظاهرة الاقتصادية يقوم على الاعتقاد بأن هناك علاقة سببية حتمية تعبر عهها القوانين الاقتصادية المراد اكتشافها، لذلك لا مجال

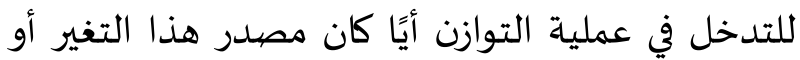
شكله، وقد رفض بعض الاقتصاديين هذا المبدأ، يقول جون ستيورات ميل (John Stuart Mill): "لا يمكن لعلم

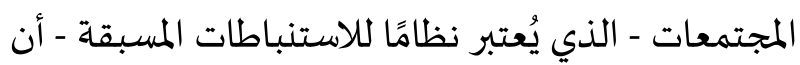
يكون علمًا للتنبؤات الإيجابية، وإنما علمًا للاتجاهات العامة فحسب" (Mill, [1827]1994: 86)، فما يوصف بالقوانين الطبيعية في العلاقات الاقتصادية كان نتاج لظروف تاريخية معينة، لذا فإنه يتغير بتغير الظروف المحيطة (Lassalle, 1904: 230)، ويرى صالح أحمد العلي (1986: 8)، بضرورة إعادة النظر في هذا الطرح بقوله:

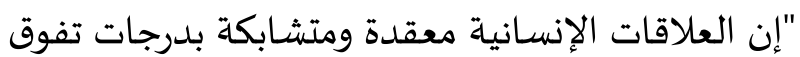

عنها قيم علمية اقتصادية علمانية، مثل : مفهوم الرشد الاقتصادي، وقيم الرجل الاقتصادي، ومبدأ الحرية المطلقة، واعتبار السوق هو أساس العلاقات الإنسانية والاجتماعية، كما أدت الى تبني المنهنج الوضعي باعتباره المنهج الوحيد

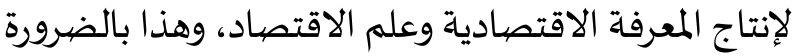
يؤدي الى الاعتقاد بوجود قوانين طبيعية يخضع لها السلوك الإنساني، الذي يتعارض مع المبدأ العقدي الإسلامي

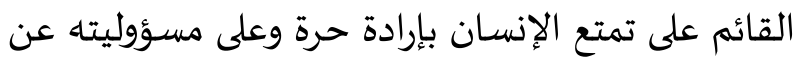
سلوكه يوم القيامة، لذلك يجب أن تكون مناهج المعرفة الاقتصادية مبنية على فلسفة المعرفة الإسلامية أو الأبستمولوجيا الإسلامية المبنية على العقيدة التوحيدية

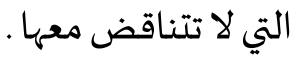

ب- توافقت المناهج الإسلامية في بناء المعرفة الاقتصادية سواء الوضعية أو التطبيقية الجزئية أو الفقهية، مع المنهج الوضعي المبني على المذهب أو الفلسفة أو العقيدة العلمانية المادية وعلى نظرتها الكونية التي تناقض الفلسفة المانية

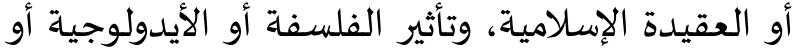

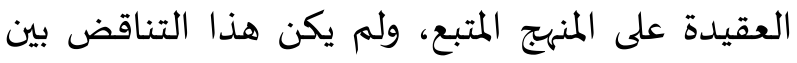
الفلسفة أو العقيدة الإسلامية والعقيدة العلمانية مركز

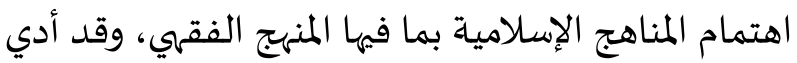

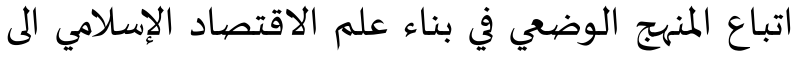
عدم تمثيل النماذج الاقتصادية للاطار النظري الذي وضعاه هذا المنهج للسلوك الاقتصادي للمجتمعات المسلمة وعدم مقدرته على دراسة المشكلات الاقتصيادياة، وتحقيق الأهداف الاقتصادية التي ولدتها قيمه الأخلاقية والدينية مثل: تحقيق التكافل الاجتماعي وعدالة توزيع الدخل والثروة ومنع أكل الأموال بالباطل التي تتم عن طريق الربا والغرر والقمار، لذلك يجب أن يكون من أهداف المناهج الإسلامية المولدة للمعرفة الاقتصادية معالجة المشاكل الاقتصادية للمجتمع المسلم. 
والثقافية والاجتماعية كمكونات أساسية تؤثر وتتأثر

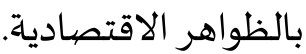

5- الحاجة إلى تعدد منهجيات التنظير لعلم الاقتصياد: إن الإخفاق المعرفي لعلم الاقتصاد نتيجة للأزمات

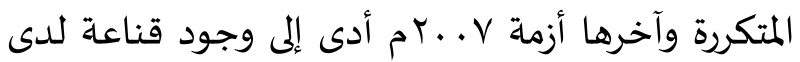
كثير من الاقتصاديين بفشل المعرفة الاقتصادية في المادية توصيف حالة الاقتصاد، وفي التنبؤ بالأزمات التي تجتاحها

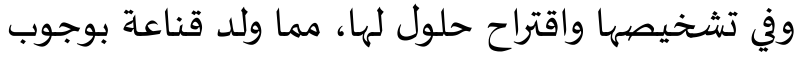
مراجعة علم الاقتصاد لاكتشاف سبب فشله. ويميل البعض إلى أن تغير التفكير الاقتصادي يستوجب مراجعة

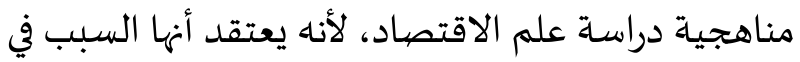
إخفاقه شيلا (Dow, Sheila, 2012)، وهذا يستوجب تغير المناهجية وفلسفة العلم (أبستمولوجيا)، أي نظرية

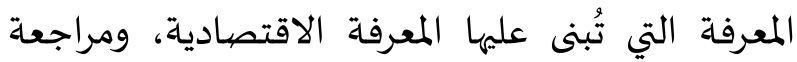
طريقة التفكير أو النظرة الكلية التي يتبناها المجتمع المعادية المعادية وهذا يستوجب مراجعة النظريات (world view)

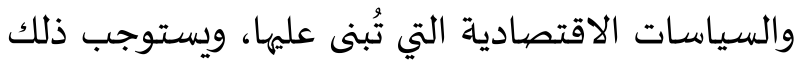

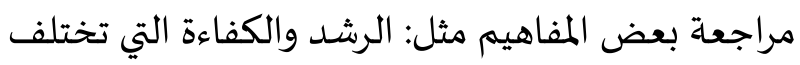

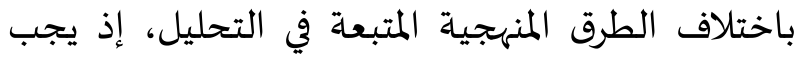
الاعتماد على النظرة الكونية التي تختلف باختلاف

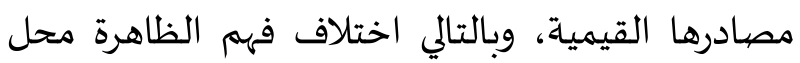
الدراسة ضمن المنظومة الثقافية للمجتمع المعين، مما يستلزم وجود تقبل للتعددية المنهجية، واختلافًا في المفاهيم، وهذا يعني الخروج من استبداد تفكير وفلسفة أحادية فرضتها المدرسة النيوكلاسيكية على التفكير المعرفي. وبصدد البحث في طرق جديدة للتفكير الاقتصادي بدأ الكُتَّاب في إعادة النظر في منهجيات مغايرة عن منهجية الفكر النيو كلاسيكي الغالب بين الاقتصاديين المعاصرين

$$
\text { مثل فكر ماركس وهايك وكينز وآدم سميث. }
$$

كثيرًا تعقد وتشابك العلاقات بين المواد الجامدة".، ذلك أن الظاهرة الاقتصادية هي ظاهرة اجتماعية لا تتحقق الا في مجتمعات وتجمعات إنسانية، والاعتقاد بوجود قوانين تحكم العلاقات الاقتصادية يعني أن الإنسان ليس له خيار، وهو مجبر على الخضيوع لتلك القوانين، وهذا يتنافى لافي التهان مع العقيدة الإسلامية في أن الإنسان مخير في سلوكاء لتاكيان الاقتصادي وهو مسؤول ومحاسب عليه، لذلك يمكن القول بأن هناك قوانين حتمية جبرية يخضع لها السلوك الاقتصادي للوحدات -كما يدعي علم الاقتصاد الإيجابي تنافي العقيدة التوحيدية التي تقوم على حرية الاختيار، وعلى مسؤولية الإنسان عن خياراته في سلوكه الاقتصادي. د- القو انين في علم الاقتصاد الإسلامي: يعتقد كثير من الاقتصاديين بما فهيه أدم سميث، مؤسس علم الاقتصاد، أن الأخلاق مؤثرة في السلوك الأك الهان

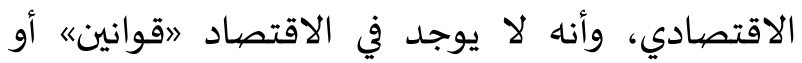

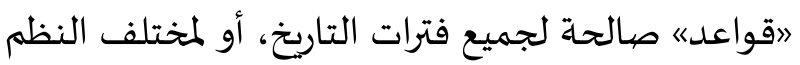
الاقتصادية" (Bairoch, 1995:224). كما أن بعض الاقتصاديين

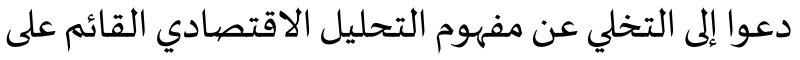
فكرة القانون الطبيعي واستبداله بدراسة العلاقة الوثيقة بين التحليل الاقتصادي والنظام الاجتماعي الذي يشكل أساس العلاقات الاجتماعية ( Heilbroner and Milberg,

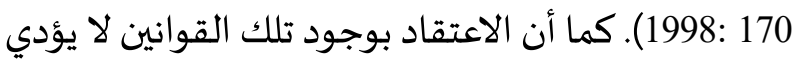
الى التعمّق في معرفة الظواهر الاقتصادية والسنن التي تحكمها والعوامل الاجتماعية والثقافية والأخلاقية والدينية التي تؤثر فهها، وبذلك لا يتحقق الهدف المعرفي لعلم الاقتصاد الذي يتوقعه المجتمع الرأسمالي وهو تنوير

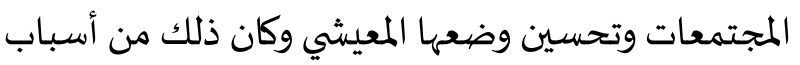
الفشل المعرفي لعلم الاقتصاد، لذلك يجب أن تبنى مناهج المعرفة لعلم الاقتصاد واعتبار القيم الأخلاقية والدينية 
و يؤدي عدم التأكد إلى تغير ذلك التفسير كلما تغيرت

$$
\text { الشواهد أو الظروف المؤثرة. }
$$

إن قبول منطق إمكانية أن يكون النظام التنظيري مفتوحاً، أي يمكن أن يتأثر بالعوامل المستجدة خانه إنهارج محددات الفكر النيوكلاسيكي، أدى إلى نشوء مدرسة

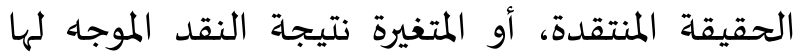

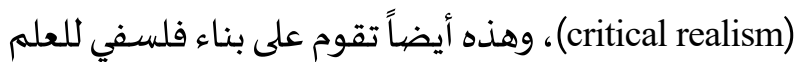
وعلى منهجية وعلى بناء نظري مختلف، ففي هذه المدرسة، فإن النظم الاجتماعية هي نظم عضوية وليست ذرية كما في الفكر النيوكلاسيكي، والحقيقة المشاهدة حسب هذه المدرسة هي نتاج الهيكلية والتركيبة والعمليات المولدة لهذه النظم، فيكون هدف العلم هو معرفة هذه التركيبة وهذه العمليات، وحيث إن هذه التركيبة هي تركيبة عضوية مترابطة؛ فإن معلوماتنا عنها تكون غير كاملة. والحقيقة التي يمكن فهمها في النظم الاجتماعية المفتوحة تكون صحيحة فقط ضمن تركيبة المنظومة الاجتماعية المحددة، لذلك يتم تقييم الرشد في هذه النظم ضمن فهم التركيبة البنائية لتلك النظم، وهذا يخالف الفكر النيوكلاسيكي أو الفكر الاقتصادي السائد بافتراض حدوث سلوك اقتصادي رشيد موحد لكل التنظيمات

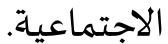

إن تغير البناء الفلسفي لعلم الاقتصاد والهدف منها ومنهجية دراستاه وبناء نظرياته في فكر كينز وفي مدرسة الحقيقة المنتقدة يستوجب تغير الأنطولوجيا (ontological) أو البناء الفكري الذي ينطلق منه البناء الفلسفي، وهذا ما لم يحدث حتى الآن، لذلك لم تستطع المنهجيات البديلة أن

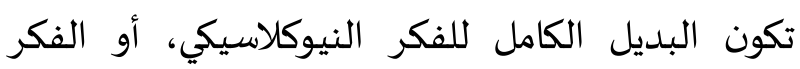
الاقتصادي السائد (mainstream economics).
إن تحليل منهاجية دراسـة علم الاقتصياد استقطب اهتمام كثير من الاقتصاديين غير التقليديين وعلى رأسهم هايك (Hayek, 1979) وروبنسن (Robinson, 1979) وكينز وللوصول إلى معايير جديدة لمنهجية (Keynes, 1921) دراسة علم الاقتصاد وللحكم على صحة نظرياته؛ open theoretical ( يستوجب اعتماد نظام نظري مفتوح

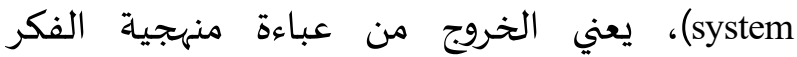
النيوكلاسيكي الذي يُعتبر أنه مالك للحقيقة وأن أي مخالف لها يكون مخطئًا ومجافيًا ، حيث اعتمد النظام النظري المفتوح للتطورات الحديثة في منهجية دراسة علم الاقتصاد خارج المنهجية التقليدية المتبعة من قبل المدرسة النيوكلاسيكية والمعتمدة على الإطار الفلسفي لكينز وفي منهجية مدرسة (الحقيقة المنتقدة) (critical realist) المرتكزة على الفلسفة الكينزية. إن فكر كينز (1921م) يقوم على بناء فلسفي للعلم، وعلى منهجية ونظرية اقتصادية مختلفة عن المدرسة النيوكلاسيكية. والمعرفة عند كينز قد تكون بديهية منطقية (tautology) وقد تكون مشاهدات تتكرر بانتظام، ولكن المعرفة عن النظم الاجتماعية يشوبها عدم التأكد، ذلك لأن النظم الاجتماعية نظام مفتوح (open systems)، والمنطقية في العلاقات الاجتماعية غير مؤكدة بسبب تعدد العوامل غير المعروفة التي تؤثر في السلوك الاجتماعي، كذلك تعاني الدراسات التطبيقية من مشكلة عدم التأكد بسبب تطور السلوك وعدم استمرارية تأثير بعض المتغيرات، مما يجعل من الصعب إعادة تكرار بعض المشاهدات بانتظام، وعليه تكون صحة نتائج الدراسات التطبيقية في تنظير العلاقات نسبية وليست مطلقة، وعدم التأكد هذا ينطبق على الاقتصاد ونظرياته، لذلك يتم اللجوء إلى المنطق لتفسير نتائج الدراسات التطبيقية، 
الوحي يعتبر المصدر الأهم لمعرفة الحقيقة المطلقة، إلى جانب العقل والمشاهدات والتجارب.

2- وضع النموذج المعرفي أو المنظور الإسلامي والقيم

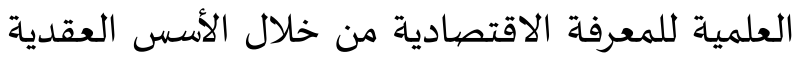

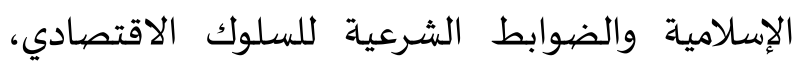
ويحدد المنظور الاقتصادي الإسلامي محل دراسـة علم الاقتصاد الإسلامي، والهدف الذي يحققه هذا العلم،

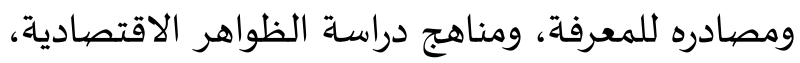
ومعايير وسائل اختبار المعرفة التي تم التوصل إليها ووسائل التحقق من المعرفة الصادقة من المعرفة الكاذبة. وفي المنظور الاقتصادي الإسلامي يتم وضع القيم العلمية الإسلامية والافتراضات الأساسية التي تُبنى عليها

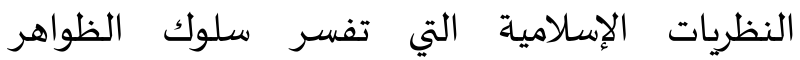
الاقتصادياة في المجتمع المسله. 3- مسح النظريات الاقتصادية التقليدية وتطوراتها، والقيم العلمياة والافتراضات الأساسية التي بُنيت عليها،

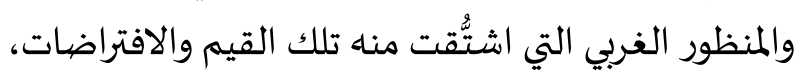

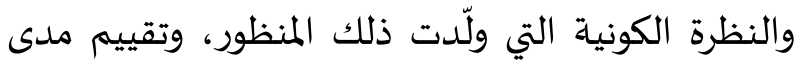

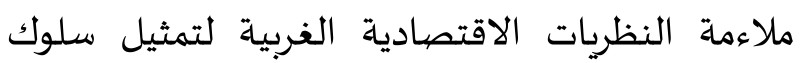

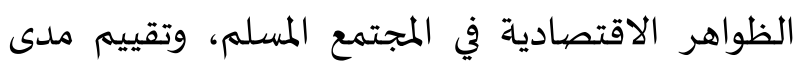

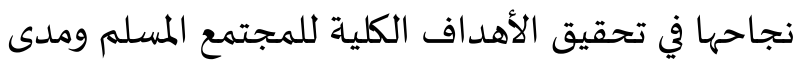
نفع الحلول التي تقترحها لحل المشكلات الاقتصادية في المجتمع المسلم، وبيان مدى تصالح أو تعارض القيم

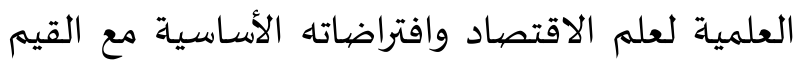
العلمية الإسلامية، مثلاً هل يمكن قبول مفهوم الرشد والعداديه

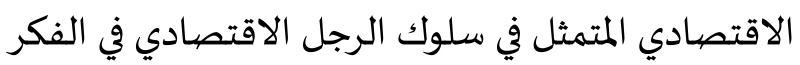
النيوكلاسيكي، أو قبول فرضية كون الإنسان أنانيًا بطبعاء

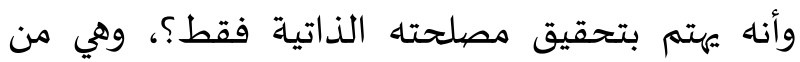
الفرضيات التي يقوم عليها البناء النظري للاقتصاد التقليدي، هل تُقبل فرضية (أن الجشع جيد) وهي فرضية لبنية

\section{6- منهج التأصيل الإسالامي للعلوم الاقتصادية:} وجدت دراسات سابقة عن إجراءات منهج التأصيل الإسلامي للعلوم الاجتماعية، والتصور لإجراءات التأصيل لمنهجية دراسـة علوم الاقتصاد الإسلامي، والتركيز هنا على الإسلى أحد العلوم الاجتماعية وهو علم الاقتصاد الإسلاهي،

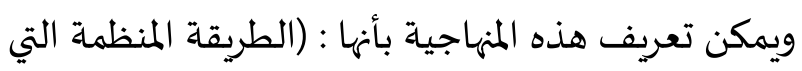

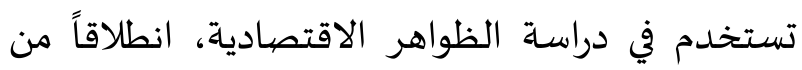

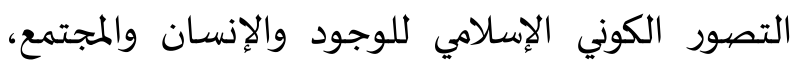
لوضع القيم العلمية والمنظور (Islamic paradigms) الاقتصادي للمجتمع المسلم الذي تُبني عليه الجهود

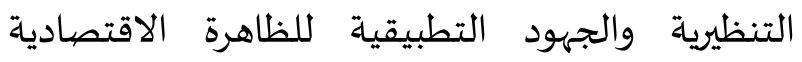
والسياسات الاقتصادية المتبعة لتحقيق الأهداف الكلية

$$
\text { للمجتمع المسلم). }
$$

ويمكن ترجمة هذا التعريف إلى الخطوات التالية: 1- وصف التصور الكوني للمجتمع، وهو تصيور

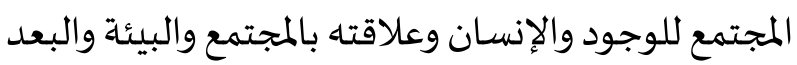
الزماني والمكاني لهذا الكون، ويجيب عن الأسئلة الوجودية ولهودية مثل: من أوجد هذا الكون؟ وكيف وُجد الكون؟ ولماذا

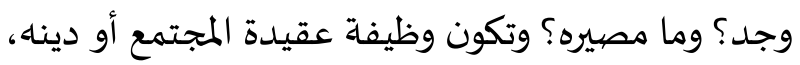
سواء الدين السماوي أو الدين البشري مثل الدين العلماني، هي الإجابة عن هذه الأسئلة. وحيث إن عقيدة

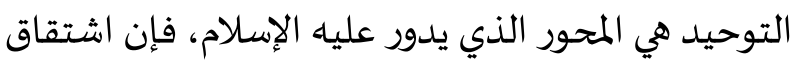

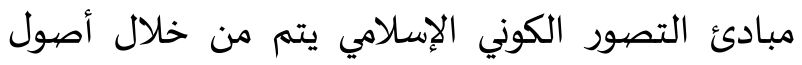
الاعتقادات الغيبية الإسلامية التي بنيت على أركان الإسلام والإيمان، وهي تشكل الأساس العقدي أو الأبستمولوجيا لنظرية المعرفة الإسلامية، وتبني نظرية المعرفة الإسلامية على هذه الأسس العقدية، التي تحدد مصادر المعرفة التي يعتمدها المجتمع العلمي المسلم لبناء المكون العلمي للعلوم بما في ذلك العلوم الاجتماعية التي منها علم الاقتصياد، وتختلف نظرية المعرفة الإسلامية عن الغربية في كون 
الإسلامية)، وتستخدم هذه النظريات لتفسير سلوك تلك

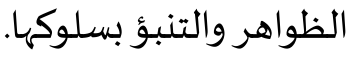

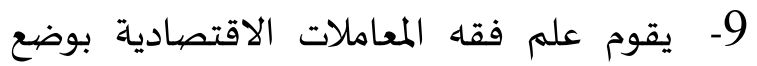
المعايير الشرعية للسلوك الاقتصادي الرشيد، وتفصيل السلوك الاقتصادي الواجب مثل: الزكاة، والسلوك المحررم مثل الربا والقمار والغرر، ووضيع الضوابط والشروط الثراد الشرعية لعقود المعاملات الاقتصادية.

10- بناء على النظرة الكونية الإسلامية يقوم المجتمع

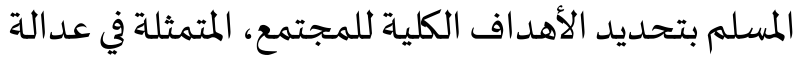
التوزيع للدخول والثروات، وتحقيق العدالة الاجتماعية والاقتصادية، وتخفيف ظاهرة الفقر، ونصب شبكات

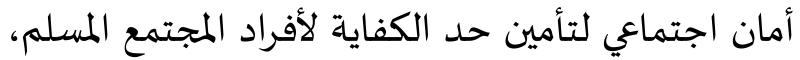
وتشغيل كل قادر على العمل، وتحقيق الاستقرار الاقتصادي

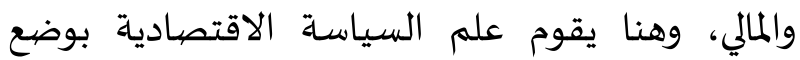
الأدوات والسياسات والإجراءات والنظم والقوانين التاني الإني تحقق هذه الأهداف، وقد تكون الأدوات لها أصل في الشريعة مثل الزكاة، والوقف، والإنفاق التطوعي، أو يكون

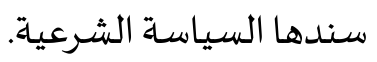

11- في دراسة علوم الاقتصاد الإسلامي يتكامل علم الاقتصاد الإسلامي، الذي يختص بالتنظير والتحليل

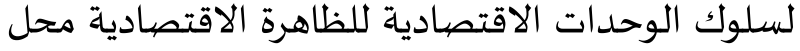

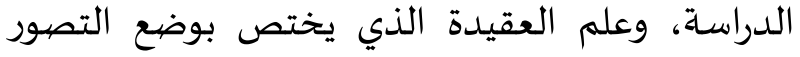
الكوني الإسلامي ووضع أبستمولوجيا أو نظرية المعرفة

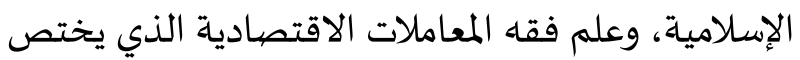

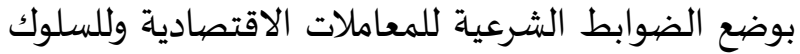
الاقتصادي الرشيد للمسلم، وعلم السياسة الاقتصادية الذي يختص بوضع السياسات الاقتصادية اللازمة لتحقيق الأهداف الاقتصادية الكلية للمجتمع وتصحيح انحرافات السلوك الاقتصادي عن السلوك الاقتصادي الرشيد للمسلم. - (المرات
تقوم عليها نظرية الإنتاج؟، هل تُقبل فرضية خضوع الظواهر الاقتصادية لقوانين حتمية لا تدخل فيها للإرادة الإلهية؟، وتقييم مدي صلاحية المنظور الاقتصادي العلماني لتمثيل النظرة الكونية الإسلامي.

6- يتم بناء المنظور الاقتصادي الإسلامي الذي يشكل

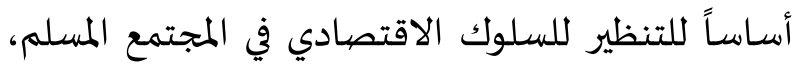
ويتم ذلك بإحلال القيم العلمية الإسلامية التي تم اشتقاقها من الأسس العقدية للمنظور الاقتصادي الإسلامي محل

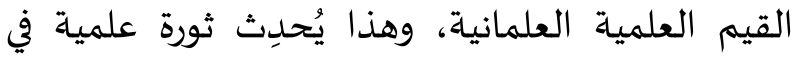
التنظير لسلوك الظواهر الاقتصادية في المجتمع المسلم. 7- يستخدم المنهج الاستنباطي لوضع معايير السلوك

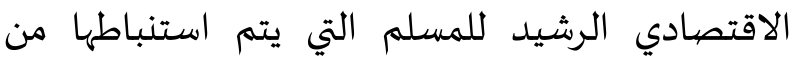

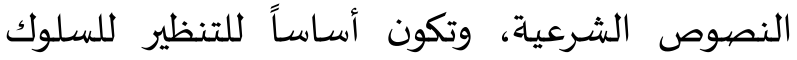
الاقتصادي للوحدات الاقتصادية للظاهرة الاقتصادية محل الدراسة في المجتمع المسلم، حيث تمثل التوجهات العقدية والضوابط الشرعية أهم المؤثرات في السلوك الهوك الاقتصادي للوحدات الاقتصادية، ويُبني علهيا السلوك الاقتصادي الرشيد لتلك الوحدات. 8- يُستخدم المنهج الاستقرائي لاختبار صحة النظريات

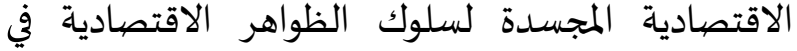
المجتمع المسلم، فإذا وُجد انحراف بين النظرية والسلوك المكان الفعلي للوحدات الاقتصادية، فإنه يعاد النظر في التفسير النيا المنطقي للسلوك الرشيد، أو في تفسير الأسس العقدية والشرعية للسلوك الرشيد للوحدات الاقتصادية لتصحيح النظرية، فإذا لم يكن هناك خلل في التنظير،

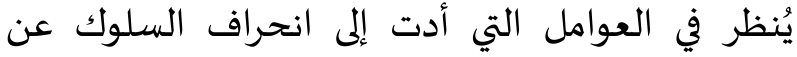

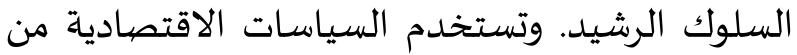

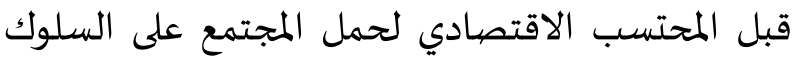
الإسلامي الرشيد، تشكل مجموع النظريات لسلوك الظواهر الاقتصادية في المجتمع المسلم (النظرية الاقتصادية 
عبدالرحيم عبدالحميد السـاعاتي دكتوراه من جامعة كولورادو بمدينة بولدر بالولايات المتحدة

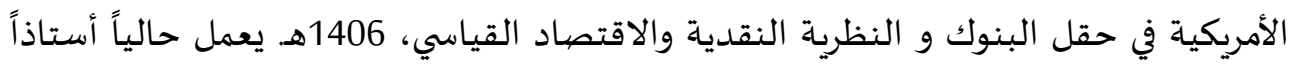
للاقتصاد والتمويل الإسلامي ومستشار بقسم الاقتصاد الإسلامي، معهد الاقتصاد الإسلامي جامعة الملك عبدالعزيز . من أهم المساهمات العلمية المنشورة. 1. الفشل المعرفي لعلم الاقتصاد، محاضرة ألقيت في سلسلة على مائدة الحوار العلمي بقسم

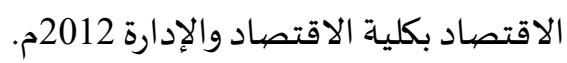

2. تشخيص الأزمة المنهجية للاقتصاد الإسلامي بحث قدم في ندوة مستقبل الاقتصاد الإسلامي

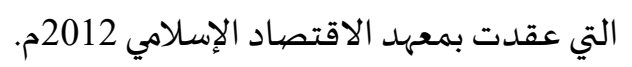

3. الحالة المعرفية لعلم الاقتصاد الإسلامية، المؤتمر العلمي الأول للأسواق المالية الناشئة:

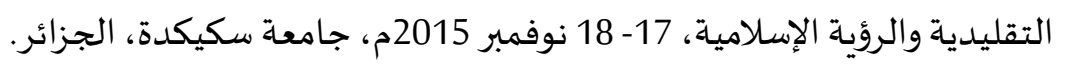

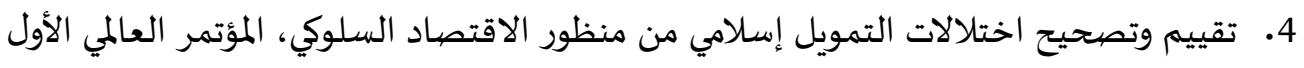

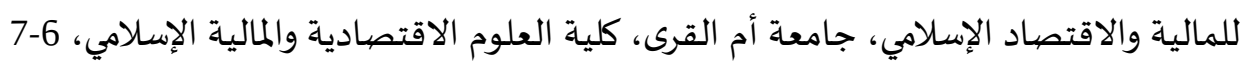
مارس 2016م. 5. نظرية المعرفة الإسلامية والقيم العلمية لعلم الاقتصاد الإسلامي: دراسـة منهجية، بحث مقدم

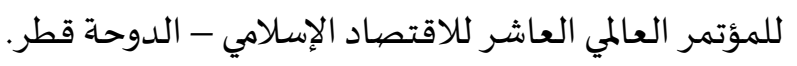
6. تأصيل البناء النظري للتمويل الاقتصادي الإسلامي: نظرية التمويل الإسلامية الحديثة، بحث مقدم للمؤتمر العلمي الدولي الثاني(البنوك الإسلامية ولتنمية الشاملة :حقوق الملكية)، جامعة العلوم الإسلامية العالمية 2019م. البريد الإلكتروني: a_alsaati@hotmail.com. 


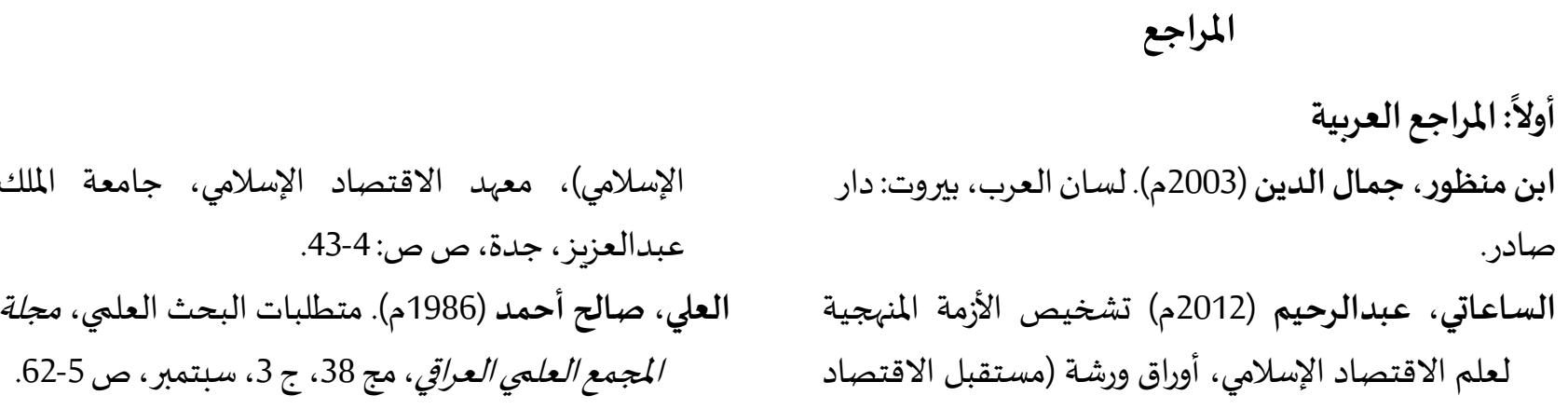

Dow, Sheila (2012) Foundation of New Economic Thinking, Palgrave Macmillan

Haneef, A. (2012) Preliminary Thoughts on Diagnosing Some Methodological Issues in Developing Islamic Economics, Workshop on Future of Islamic Economics, IEI, KAU, Jeddah, pp: 133-153

Hayek, F.A.(1979) The Counter Revolution of Science, 2ed, Indianapolis :liberty Press

Heilbroner, Robert (1996) The Embarrassment of Economics, Challenge, Vol. 39, No. 6, pp. 46-49.

Keynes, J.M. (1921) A Treaties on Propability, Dover Phoenix Edition Lassalle, 1904: 230) Lassalle, Ferdinand (1904). Capital et travail, Paris: V. Giard et $\mathrm{E}$. Brière.

Mannheim, Karl ([1929]1956). Idéologie et utopie, Paris: Rivière

\section{Translation of Arabic References}

Al-Ali, Salah Ahmad (1986). Mutatalabāt al-Bahth al'Ilmī [The Requirements of the Scientific Research], Majalat al-Majma' al-'Ilmī al-Irāqū, Vol. 38, No. 3, September, pp. 5-62.

Alsaati, A. (2012), Diagnoses Islamic Economics Methodology ,proceeding of ( The Future of Islamic economics) I.E.I, King Abdulaziz University, pp: 4-43.
Max Weber (1993) Basic concept of Sociology, New York: Citadel Press, P. 56.

Mill, [1827]1994: 86) Mill, John Stuart ([1827]1994) The Logic of the Moral Sciences, London: Open Court Publishing

Nienhaus, V. (2012) Method and Substance of Islamic Economics: Moving Where? Workshop on Future of Islamic Economics, IEI, KAU, Jeddah, pp: 167-197.

Robinson, A. (1979), Collected Economic Papers Vol. v p.110,(Oxford: Basil Blackwell ).

Schumpeter, Joseph A. (1949) Science and Ideology, The American Economic Review, Vol. 39, No. 2 (March), pp. 346-359.

The American Heritage Dictionary (2009) 4th ed. Houghton Miffin Company.

Ibn Mandhūr, Jamāl Dīn (2003). Lisān al-'Arab [Classical Arabic Dictionary], Beirut: Dar Sader. 


\title{
The Islamic Methodology for Islamic Economics Methods
}

\author{
Abdulrahim Abdulhamid Al-Saati \\ Prof. Dr. of Islamic Economics and Finance \\ Islamic Economics Institute, King Abdulaziz University, Jeddah
}

\begin{abstract}
The absence of Islamic concept of methodology, which is driven from epistemology or Islamic philosophy of knowledge i.e. Islamic faith, in building process of Islamic economic knowledge, resulted in adapting methods in producing Islamic economic sciences which are based on scholar methodology, which is contradicting Islamic faith, this paper call for adapting methods which are based on Islamic faith in producing Islamic economic sciences, and suggested method to can be used to rewrite Islamic economics on Islamic methodology.
\end{abstract}

KEYWORDS: methods, methodology, epistemology, paradigms.

JEL CLASSIFICATION: B410, B590

KAUJIE CLASSIFICATION: H21 